\title{
Capillary fracture of soft gels
}

\author{
Joshua B. Bostwick* \\ Department of Mathematics, North Carolina State University, Raleigh, North Carolina 27695, USA \\ Karen E. Daniels \\ Department of Physics, North Carolina State University, Raleigh, North Carolina 27695, USA \\ (Received 23 July 2013; published 28 October 2013)
}

\begin{abstract}
A liquid droplet resting on a soft gel substrate can deform that substrate to the point of material failure, whereby fractures develop on the gel surface that propagate outwards from the contact line in a starburst pattern. In this paper, we characterize (i) the initiation process, in which the number of arms in the starburst is controlled by the ratio of the surface tension contrast to the gel's elastic modulus, and (ii) the propagation dynamics showing that once fractures are initiated they propagate with a universal power law $L \propto t^{3 / 4}$. We develop a model for crack initiation by treating the gel as a linear elastic solid and computing the deformations within the substrate from the liquid-solid wetting forces. The elastic solution shows that both the location and the magnitude of the wetting forces are critical in providing a quantitative prediction for the number of fractures and, hence, an interpretation of the initiation of capillary fractures. This solution also reveals that the depth of the gel is an important factor in the fracture process, as it can help mitigate large surface tractions; this finding is confirmed with experiments. We then develop a model for crack propagation by considering the transport of an inviscid fluid into the fracture tip of an incompressible material and find that a simple energy-conservation argument can explain the observed material-independent power law. We compare predictions for both linear elastic and neo-Hookean solids, finding that the latter better explains the observed exponent.
\end{abstract}

DOI: 10.1103/PhysRevE.88.042410

PACS number(s): 68.03.Cd, 46.50.+a, 46.25.-y, 46.35.+z

\section{INTRODUCTION}

The interaction of soft substrates with fluid interfaces is common to many biological, medical, and industrial processes. For this reason, the field of elastocapillarity [1], in which one studies how surface tension forces couple to the deformations of elastic substrates, has been the subject of much recent attention. Even the most basic characterization of the wetting forces at the three-phase contact line remains in dispute, especially for soft viscoelastic materials [2] that have both liquid and solid properties. For such materials, the Young-Dupré law for a liquid wetting a hard solid, Neumann's law for a liquid wetting another liquid, or some hybrid thereof may apply [3,4]. Theoretical efforts have been made to bridge the gap between the hard solid and the liquid regime, such as introducing the concept of solid surface tension [5,6]. Alternative methods employ computational approaches such as density functional theory [7] and molecular dynamic simulations [4] to gain a more thorough understanding of the wetting forces acting at the contact line.

In general, the elastic resistance must be comparable in magnitude to the surface tension forces applied to the elastic medium for many elastocapillary phenomenon. The elastocapillary number $\sigma /(E L)$ is a typical measure of the relative importance of capillarity to elasticity. Here $\sigma$ is the surface tension, $E$ the elastic modulus, and $L$ a characteristic length scale. In experiments, it is typically easiest to adjust $L$, as seen in the wrinkling of elastic sheets [8-10], capillary origami [11], and buckling of elastic fibers [12]. However, it is also possible to use a gel as the solid phase [2,5,13,14], which permits $E$ to be tuned over several orders of magnitude.

*Corresponding author: joshua.bostwick@ northwestern.edu
We focus our attention on recent experiments in which surfactant-laden fluid droplets are applied to soft gel substrates [14-16], as shown in Fig. 1. In these experiments, a partially wetting liquid spreading on an agarose or gelatin substrate can produce capillary fractures that originate at the contact line and propagate outwards in a starburst pattern. It is convenient to divide the fracture process into three phases: (i) initiation, (ii) nucleation, and (iii) propagation. The initiation phase sets a critical wave number through the elastic deformation field that controls the number of arms within the starburst fracture. In previous experiments, the number of arms has been shown to be controlled by the ratio of surface tension contrast to the gel's elastic modulus [14]. The nucleation process in soft materials such as agarose gel is not deterministic; rather, thermal fluctuations begin the fracture dynamics after some finite time delay $[17,18]$. Once the cracks are nucleated, they fill with fluid from the droplet and grow with a universal power law (a)

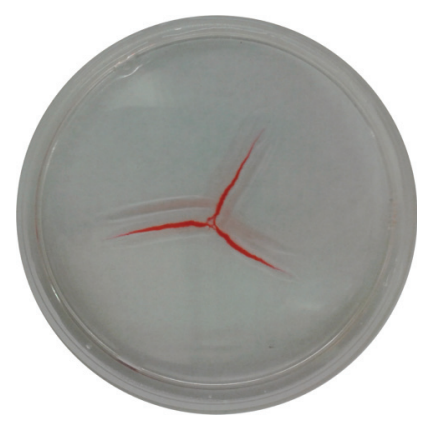

(b)

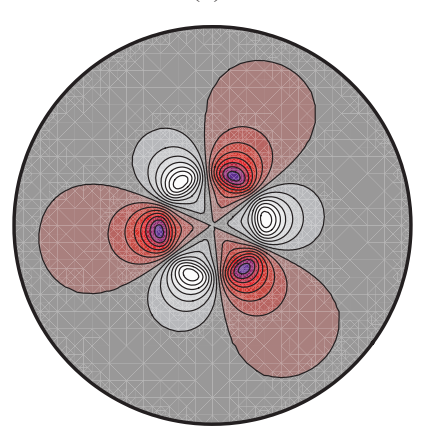

FIG. 1. (Color online) (a) A starburst fracture, formed by placing a droplet of liquid at the center of a petri dish containing agarose gel. (b) Computed displacement field, with $n=3$ arms, induced by the wetting forces from the droplet. 


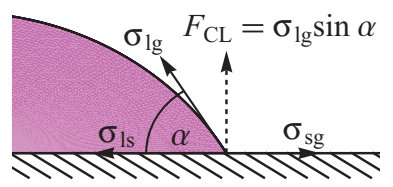

FIG. 2. (Color online) The Young-Dupré equation, (1), shown schematically as a horizontal force balance, as well as the unbalanced (vertical) contact-line force $F_{\mathrm{CL}}$.

$L \propto t^{3 / 4}$, which does not scale with any material parameters [14]. Intriguingly, even the application of a superspreading surfactant (Silwet 77) does not increase the exponent $[15,16]$. In this paper, we analyze the initiation and propagation processes separately.

To study problems coupling elasticity to capillarity, it is helpful to put the typical length scales involved with such deformations into perspective. For a liquid on a hard substrate, the wetting properties are defined by the YoungDupré equation [19,20],

$$
\sigma_{s g}-\sigma_{l s}=\sigma_{l g} \cos \alpha,
$$

which relates the liquid-gas $\sigma_{l g}$, liquid-solid $\sigma_{l s}$, and solid-gas $\sigma_{s g}$ surface tensions to the static contact angle $\alpha$. Figure 2 illustrates the interpretation of the Young-Dupré relationship as a horizontal force balance. Note that this formulation also leads to an imbalance of vertical forces with magnitude $F_{\mathrm{CL}}=$ $\sigma_{l g} \sin \alpha$. For a soft substrate with elastic modulus $E$, this force gives rise to deformations of size $\ell \sim \sigma_{l g} / E$, more commonly referred to as the elastocapillary length. For example, water $\left(\sigma_{l g}=72 \mathrm{mN} / \mathrm{m}\right)$ wetting a glass substrate $(E=70 \mathrm{GPa})$ yields a characteristic deformation $\ell \sim 10^{-12} \mathrm{~m}$, justifying the neglect of the unbalanced force. Recently, Jerison et al. have used fluorescence confocal microscopy to show that water droplets interacting with silicone gel $(E=3 \mathrm{kPa})$ yield micron-size substrate deformations, $\ell \sim 10^{-6} \mathrm{~m}[5,6]$. For the capillary fracture experiments shown in Fig. 1, which involve ultrasoft agaraose substrate $(E \sim 10 \mathrm{~Pa})$, the elastocapillary length corresponds to $\ell \sim 10^{-3} \mathrm{~m}$ (millimetric) deformations. Given the size of such deformations, it is therefore unsurprising that fractures occur.

We begin Sec. II by defining the equations governing the deformation of an elastic substrate loaded by a partially wetting liquid droplet with a corrugated contact line. We solve the governing equations to show that there is a critical disturbance with wave number $n_{c}$ that generates the largest elastic response, as measured by the tangential displacement, which we associate with the number of arms in a starburst fracture. The solution illustrates that the location $R$ of the unbalanced contact-line force is an important parameter in wave-number selection. This dependence is quantitatively confirmed in experiments where the wetting conditions $\alpha$ are fixed and the volume $V$ is varied, validating our interpretation of the initiation of capillary fractures. In addition, the depth of the gel can play a similar role in the initiation process, which we confirm with experiments. Section III briefly discusses the nucleation process. In Sec. IV, we model the crack propagation dynamics by considering the transport of an inviscid fluid into the crack tip of an elastic solid, subject to energy conservation (Bernoulli's law). While it is reasonable to assume that the solid behaves as a linear elastic material for the deformation
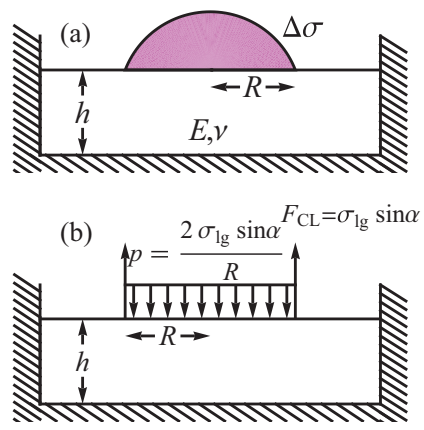

FIG. 3. (Color online) Definition sketch: schematic of (a) the liquid droplet wetting an elastic substrate and (b) the associated wetting forces included in the model.

phase, this assumption is almost certainly violated once a fracture has been nucleated. Therefore, we develop a model for the propagation dynamics which allows for considering either (i) a linear elastic or (ii) a neo-Hookean solid. Both elastic models for the solid predict power law growth, but the exponent from the neo-Hookean model matches the observed power law behavior $L(t) \propto t^{3 / 4}$. The predicted exponent and prefactor do not depend on any of the material parameters, consistent with experimental observations [14-16]. We conclude with some remarks in Sec. V regarding capillary fracture in soft materials.

\section{CRACK INITIATION}

Consider a liquid droplet, contained at its free surface by the liquid-gas surface tension $\sigma_{l g}$ and resting on a linear elastic substrate of thickness $h$, which is characterized by an elastic modulus $E$ and Poisson ratio $v$, as shown in Fig. 3(a). This partially wetting liquid interacts with the solid through the capillary pressure $p=2 \sigma_{l g} \sin \alpha / R$ uniformly distributed over the liquid-solid contact area and the unbalanced (vertical) contact-line force $F_{\mathrm{CL}}=\sigma_{l g} \sin \alpha$ applied at the contact-line radius $R$ [see Fig. 3(b)]. Here we note that an alternative model of wetting, which, in addition to the capillary pressure and vertical contact-line force, also includes a horizontal contact-line force directed into the liquid phase, has recently been put forth $[3,4]$. For the purposes of this paper, however, we restrict ourselves to a model of wetting that includes the capillary pressure and vertical contact-line force. The extent to which the liquid partially wets the solid is controlled by the surface tension contrast $\Delta \sigma=\sigma_{s g}-\sigma_{l s}$ or, equivalently, the static contact angle $\alpha$, defined by the Young-Dupré equation [Eq. (1)]. We characterize the elastic response in the substrate due to both the capillary pressure and the contact-line force associated with a corrugated contact line.

\section{A. Field equations}

We begin by introducing the displacement field $\boldsymbol{u}$,

$$
\boldsymbol{u}=u_{r}(r, \theta, z) \hat{\boldsymbol{e}}_{r}+u_{\theta}(r, \theta, z) \hat{\boldsymbol{e}}_{\theta}+u_{z}(r, \theta, z) \hat{\boldsymbol{e}}_{z},
$$

in cylindrical coordinates $(r, \theta, z)$, which satisfies the governing elastostatic Navier equations,

$$
(1-2 v) \nabla^{2} u+\nabla(\nabla \cdot u)=0 .
$$

The strain field $\boldsymbol{\varepsilon}$ is defined as

$$
\boldsymbol{\varepsilon}=\frac{1}{2}\left(\nabla \boldsymbol{u}+(\nabla \boldsymbol{u})^{t}\right)
$$


while the stress field $\sigma_{i j}$ for this linear elastic solid is given by

$$
\sigma_{i j}=\frac{E}{1+v}\left(\varepsilon_{i j}+\frac{v}{1-2 v} \varepsilon_{k k}\right) .
$$

\section{B. Boundary conditions}

We assume the elastic substrate is pinned to a rigid support at $z=0$ by enforcing a zero displacement boundary condition there:

$$
\boldsymbol{u}(r, \theta, 0)=0 .
$$

In experiments this support corresponds to the bottom of the dish holding the substrate material. Similarly, we specify the surface tractions on the free surface, $z=h$,

$$
\begin{gathered}
\sigma_{z z}(r, \theta, h)-\Sigma_{s} \nabla_{\|}^{2} u_{z}(r, \theta, h)=F(r, \theta), \\
\sigma_{r z}(r, \theta, h)=\sigma_{\theta z}(r, \theta, h)=0,
\end{gathered}
$$

where $\nabla_{\|}^{2}$ is the surface Laplacian and $F(r, \theta)$ is the applied force associated with the liquid-solid (wetting) interactions. Here we introduce the solid surface tension $\Sigma_{s}$ for rigor as well as to regularize the singularity associated with applying a $\delta$ function force to the surface of an elastic medium, as discussed in [6].

We now develop a model for the forces $F(r, \theta)$ associated with the wetting of a liquid droplet on a soft elastic substrate. First, we note that the surface of our gel substrate is heterogeneous so that the location of the contact line generally takes the form $r=R(\theta)$ (in fact, corrugations are typically present even for ideal solid or liquid substrates). For a liquid droplet held by uniform surface tension $\sigma_{l g}$, the wetting forces associated with this configuration are given by

$$
F(r, \theta)=-p H(R(\theta)-r)+F_{\mathrm{CL}} \delta(r-R(\theta)),
$$

where $H$ and $\delta$ are the Heaviside and $\delta$ functions, respectively [see Fig. 3(b)]. Here the capillary pressure $p=2 \sigma_{l g} \sin \alpha / R$ is uniformly distributed over the liquid-solid surface area, whereas the unbalanced contact-line force $F_{\mathrm{CL}}=\sigma_{l g} \sin \alpha$ is applied as a point load at the contact line. Note the orientation of the applied forces; the capillary pressure $p$ compresses the substrate, while the contact-line force $F_{\mathrm{CL}}$ tends to pull the substrate upwards. As we are interested in identifying a critical wave number to associate with the number of arms in the starburst fracture, we assume that the contact-line radius has the representation $R(\theta)=R(1+\epsilon \cos n \theta)$, where $n$ is the azimuthal wave number. We then apply this functional form for $R(\theta)$ to Eq. (8) and expand for small $\epsilon \ll 1$ to show that the nonuniform $O(\epsilon)$ part of the wetting force is given by

$$
F(r, \theta)=\left(-p H^{\prime}(R-r)+F_{\mathrm{CL}} \delta^{\prime}(r-R)\right) R \cos n \theta .
$$

Equations (3), (6), (7) and (9) define a boundary value problem governing the elastic deformations of a substrate interacting with a liquid droplet with a corrugated contact line.

\section{Displacement potential}

We simplify the elastostatic governing equations, (3), by introducing the displacement potentials $\boldsymbol{G}$ and $\boldsymbol{A}$ defined such that

$$
\boldsymbol{u}=\frac{1+v}{E}\left(2(1-v) \nabla^{2} \boldsymbol{G}-\nabla(\nabla \cdot \boldsymbol{G})+\nabla \times \boldsymbol{A}\right),
$$

with

$$
\boldsymbol{G}=\xi(r, \theta, z) \hat{\boldsymbol{e}}_{z}, \quad \boldsymbol{A}=2 \psi(r, \theta, z) \hat{\boldsymbol{e}}_{z} .
$$

Substituting (10) into the coupled system of differential equations, (3), delivers a set of uncoupled equations for $\xi, \psi$ :

$$
\nabla^{4} \xi=0, \quad \nabla^{2} \psi=0 .
$$

The displacement, (6), and traction, (7), boundary conditions can similarly be written in terms of the potential functions $\xi, \psi$.

\section{Fourier-Hankel expansion}

We seek solutions to (12) for the potential functions using Fourier-Hankel transforms. To begin, we assume that $\xi$ and $\psi$ have the following Fourier decomposition:

$$
\begin{gathered}
\xi(r, \theta, z)=\sum_{n=0}^{\infty} \xi_{n}(r, z) \cos n \theta, \\
\psi(r, \theta, z)=\sum_{n=0}^{\infty} \psi_{n}(r, z) \sin n \theta .
\end{gathered}
$$

Next, the Hankel transform is applied to each Fourier component $\xi_{n}(r, z), \psi_{n}(r, z)$ to yield

$$
\begin{aligned}
& \hat{\xi}_{n}(s, z)=\int_{0}^{\infty} r \xi_{n}(r, z) J_{n}(s r) d r, \\
& \hat{\psi}_{n}(s, z)=\int_{0}^{\infty} r \psi_{n}(r, z) J_{n}(s r) d r,
\end{aligned}
$$

where $J_{n}$ is the Bessel function of the first kind and $s$ is the radial wave number. One applies the inverse Hankel transform,

$$
\begin{aligned}
& \xi_{n}(r, z)=\int_{0}^{\infty} s \hat{\xi}_{n}(s, z) J_{n}(s r) d s, \\
& \psi_{n}(r, z)=\int_{0}^{\infty} s \hat{\psi}_{n}(s, z) J_{n}(s r) d s,
\end{aligned}
$$

to recover $\xi_{n}, \psi_{n}$ and, equivalently, $\xi, \psi$.

\section{E. Reduced equations}

The following dimensionless variables are introduced:

$$
\begin{aligned}
u & \equiv \hat{u} \frac{\sigma_{l g} \sin \alpha}{E}, \quad r \equiv \hat{r} h, \quad z \equiv \hat{z} h, \\
s & \equiv \frac{\hat{s}}{h}, \quad R \equiv \hat{R} h .
\end{aligned}
$$

with lengths scaled by the thickness of the elastic substrate $h$ and elastic deformations by the elastocapillary length $\ell \equiv$ $\frac{\sigma_{l g} \sin \alpha}{E}$. In the derivation that follows, we drop the hats for notational simplicity. Substituting the expansions (13), (14) into (12) gives reduced field equations for $\hat{\xi}_{n}, \hat{\psi}_{n}$,

$$
\begin{aligned}
\nabla_{n}^{4} \hat{\xi}_{n} & =\left(\frac{d^{2}}{d z^{2}}-s^{2}\right)^{2} \hat{\xi}_{n}=0, \\
\nabla_{n}^{2} \hat{\psi}_{n} & =\left(\frac{d^{2}}{d z^{2}}-s^{2}\right) \hat{\psi}_{n}=0 .
\end{aligned}
$$


The following conditions are enforced on the rigid support $z=0$,

$$
\begin{array}{r}
\frac{d \hat{\xi}_{n}}{d z}=0, \quad \hat{\psi}_{n}=0, \\
(1-2 v) \frac{d^{2} \hat{\xi}_{n}}{d z^{2}}-2(1-v) s^{2} \hat{\xi}_{n}=0,
\end{array}
$$

while on the free surface $z=1$ we require

$$
\begin{aligned}
& v \frac{d^{3} \hat{\xi}_{n}}{d z^{3}}+(1-v) s^{2} \frac{d \hat{\xi}_{n}}{d z}=0, \quad \frac{d \hat{\psi}_{n}}{d z}=0, \\
& (1-v) \frac{d^{3} \hat{\xi}_{n}}{d z^{3}}-(2-v) s^{2} \frac{d \hat{\xi}_{n}}{d z}+\Upsilon(1+v) s^{2} \\
& \quad \times\left((1-2 v) \frac{d^{2} \hat{\xi}_{n}}{d z^{2}}-2(1-v) s^{2} \hat{\xi}_{n}\right)=\hat{F}_{n},
\end{aligned}
$$

with

$$
\hat{F}_{n}(s)=\Lambda\left((n-3) J_{n}(\Lambda s)-\Lambda s J_{n-1}(\Lambda s)\right) .
$$

The following dimensionless groups arise naturally from this choice of scaling,

$$
\Upsilon \equiv \frac{\Sigma_{s}}{E h} \quad \text { and } \quad \Lambda \equiv \frac{R}{h} .
$$

Here $\Upsilon$ is the solid elastocapillary number and $\Lambda$ is the aspect ratio or dimensionless contact-line radius. The solution to (17)-(19) is given by

$$
\begin{aligned}
\hat{\xi}_{n}= & C_{n}\left(\cosh (s z)+\frac{s z \sinh (s z)}{2(1-2 v)}\right) \\
& +D_{n}(s z \cosh (s z)-\sinh (s z)), \quad \hat{\psi}_{n}=0,
\end{aligned}
$$

where

$$
\begin{aligned}
C_{n} & =\frac{4 \hat{F}_{n}(s)(2 v-1)(s \cosh (s)+(2 v-1) \sinh (s))}{s^{3}\left(5+2 s^{2}-12 v+8 v^{2}+(3-4 v) \cosh (2 s)+2 s \Upsilon\left(1-v^{2}\right)((3-4 v) \sinh (2 s)-2 s)\right)}, \\
D_{n} & =\frac{2 \hat{F}_{n}(s)(s \sinh (s)+2(1-v) \cosh (s))}{s^{3}\left(5+2 s^{2}-12 v+8 v^{2}+(3-4 v) \cosh (2 s)+2 s \Upsilon\left(1-v^{2}\right)((3-4 v) \sinh (2 s)-2 s)\right)} .
\end{aligned}
$$

We compute the Fourier components $\xi_{n}, \psi_{n}$ in real space by evaluating the inverse Hankel transform, (15). Once the potential functions $\xi, \psi$ are known, the displacement $\boldsymbol{u}$, strain field $\varepsilon$, and stress field $\sigma$ are obtained via substitution into (10), (4), and (5), respectively.

\section{F. Results}

We compute the elastic response in the substrate for a fixed wave number $n$, consistent with the boundary conditions (6) and (7). That is, we assume that a corrugated contact line, with wave number $n$, gives rise to surface tractions, (7), that generate displacements $\boldsymbol{u}$-and, equivalently, stresses $\boldsymbol{\sigma}$ and strains $\boldsymbol{\varepsilon}$-within the gel substrate. We seek to develop a criterion based upon our solution for the elastic fields, which will yield a prediction for the critical wave number $n_{c}$ that is consistent with the number of fractures seen in experiments. This value can then be compared with the number of fracture arms observed in experiments.

For the problem considered here, there are three independent components of the displacement field, six for the strain field, and six for the stress field, all of which vary in the two-dimensional $(r, z)$ space (domain) in cylindrical coordinates. We utilize several experimental observations to formulate our criteria. First, we restrict our criteria to components of the displacement field, as failure in agarose gels typically corresponds to disentanglement of the helices within the network of cross-linked, polymeric bonds. Second, experiments reveal that fractures are typically of the Mode I (opening) variety [14]. These observations suggest that our "failure criterion" should correlate with the maximum value of the $u_{\theta}$ component of the displacement field. Accordingly, we compute the displacement $u_{\theta}(r, z)$ for fixed values of the parameters $(\Lambda, \Upsilon, n, \nu)$. We simplify our problem further by setting the Poisson ratio $v=1 / 2$, which is a reasonable assumption for incompressible agarose gels. In this case, the elastic modulus is then simply related to the shear modulus through the relationship $E=3 G$.

The connection between the problem considered here and the experiment in question is somewhat subtle. For a given set of experimental conditions, one defines the liquid-solid (wetting) interactions by the choice of fluid, which sets (equivalently) either the surface tension contrast $\Delta \sigma$ or the static contact angle through the relation $\Delta \sigma / \sigma_{l g}=\cos \alpha$. Hence, the unbalanced contact-line force $F_{\mathrm{CL}}=\sigma_{l g} \sin \alpha$ could also be written in terms of the surface tension contrast $\Delta \sigma$, if desired. Note that increasing the surface tension contrast $\Delta \sigma$ is equivalent to decreasing the static contact angle $\alpha$ and consequently the magnitude of the contact-line force $F_{\mathrm{CL}}$. Naively, one might think that fixing the droplet volume $V$, as is typically done in experiments, would set the contact-line radius $R$ and hence the location of the contact-line force. However, $R$ is also a function of the the wetting conditions. For a spherical-cap droplet, this relationship is given by

$$
R^{3}=\frac{3 V}{\pi}\left(\frac{\sin ^{3} \alpha}{2-3 \cos \alpha+\cos ^{3} \alpha}\right),
$$

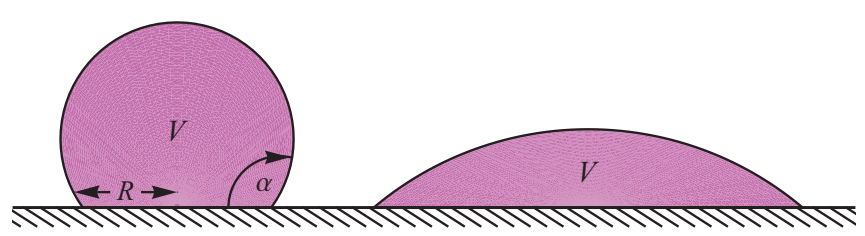

FIG. 4. (Color online) Schematic of the relationship between the contact-line radius $R$ and static contact angle $\alpha$ for two spherical-cap droplets of identical volume $V$. 
(a)

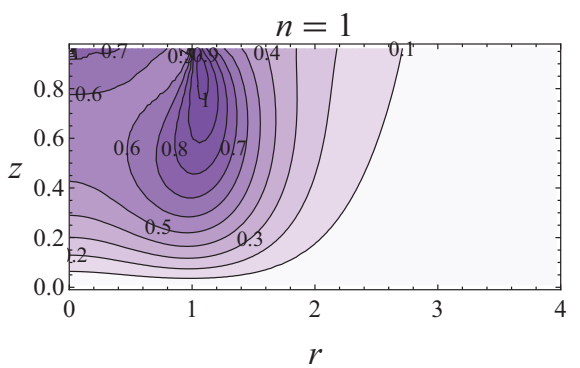

(b)

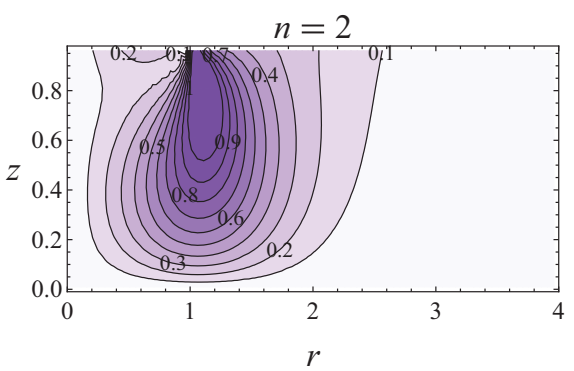

(c)

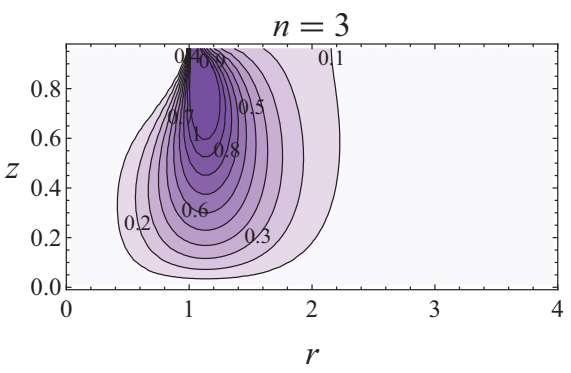

FIG. 5. (Color online) Tangential displacement $u_{\theta}$ in the $(r, z)$ plane for wave number (a) $n=1$, (b) $n=2$, and (c) $n=3$ with surface tractions applied at contact-line radius $\Lambda=1$ with $\Upsilon=0.01$.

as shown schematically in Fig. 4. The difficulty is that adjusting $\Delta \sigma$ for fixed $V$, as is typically done in experiments, changes both $R$ and $\alpha$. The benefit is that it is possible to explore a wide range of contact-line radii $R$ by changing either $V$ or $\Delta \sigma$.

To summarize, both the magnitude $F_{\mathrm{CL}}$ and the location $R$ of the contact-line force implicitly depend upon the wetting conditions through the static contact angle $\alpha$. Henceforth, we restrict ourselves to variations in $R$ and, equivalently, $\Lambda$. To isolate the critical disturbance $n_{c}$, we fix the parameters $\Lambda, \Upsilon$ and compute the displacement $u_{\theta}$ everywhere in the $(r, z)$ plane for each wave number $n$, searching for its maximum value, our failure criterion. As such, Fig. 5 shows that the location of maximum displacement occurs directly beneath the contact line $\Lambda=1$ at some finite depth below the gel surface $z=1$. For this set of parameters $\Lambda=1, \Upsilon=0.01$, the critical disturbance is the $n=2$ mode, as it generates the greatest elastic response in the gel substrate [see Fig. 6(a)].

We systematically study the parametric dependence of the critical disturbance upon the parameters $\Lambda, \Upsilon$ utilizing the observation that the maximum displacement occurs directly beneath the contact line. Figure 6 plots the displacement $u_{\theta}$ as a function of the depth $z$ for various wave-number $n$ disturbances to demonstrate that the critical disturbance depends strongly upon the contact-line radius $\Lambda$. For example, Fig. 6(a) shows that the $n=1$ mode is the critical disturbance for $\Lambda=1 / 2$, whereas the $n=3$ mode is the most dangerous for $\Lambda=3 / 2$, as shown in Fig. 6(c). In general, one observes in Fig. 6 that the critical disturbance is the one that penetrates deepest into the elastic layer.

For a fixed wave number $n$, we compute the maximum displacement $u_{m}$ through the depth of the elastic layer as a function of the contact-line radius $\Lambda$, as shown in Fig. 7 for two solid elastocapillary numbers, (a) $\Upsilon=0.1$ and (b) $\Upsilon=1$. For a given $\Lambda$, there is a wave number $n_{c}$ that generates the largest elastic response, as measured by $u_{\theta}$. Here one should note the tight spacing between curves in Fig. 7, which perhaps explains why a range of wave numbers is observed for a given experiment. More specifically, slight variations in $\Lambda$ due to a number of experimental factors can alter the expected wave number. Figure 8 plots the critical wave number $n_{c}$ against the contact-line radius $\Lambda$. Here we note that our theoretical prediction is consistent with experiments for fixed-volume droplets [14], which indicate that the number of arms in the starburst fracture increases with increasing (decreasing) $\Delta \sigma(\alpha)$ and, equivalently, increasing $\Lambda$. In fact, Fig. 9 shows a good quantitative agreement between theory and experiments for fixed-volume droplets. For comparison, we have estimated experimental $\Lambda$ values using reasonable values of the liquid-solid surface tension $\sigma_{l s}$ to compute the contact angle $\alpha$ and, equivalently, $R$.

Our model indicates that it is the location $\Lambda$ of the contactline force that selects the critical wave number $n_{c}$, as opposed to the magnitude of that force. In fact, the magnitude of the contact-line force, $F_{\mathrm{CL}} / \sigma_{l g}=\sin \alpha$, decreases with increasing (decreasing) $\Delta \sigma(\alpha)$, which is somewhat paradoxical considering that naively one expects less fractures for smaller forces. Our interpretation of the fracture mechanism is confirmed in Fig. 10, which plots the experimentally observed wave number against $V^{1 / 3}$ for fixed $\alpha$ compared to the model's prediction. We observe quantitative agreement between the model and experiments.

Our computations reveal that the thickness $h$ of the gel substrate is also a critical parameter in determining the critical wave number, as $\Lambda \equiv R / h$ couples two length scales, $R$ and $h$. (a)

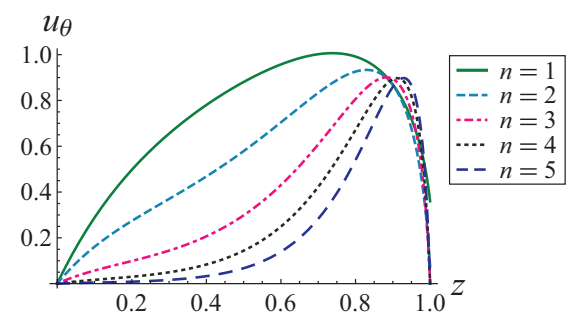

(b)

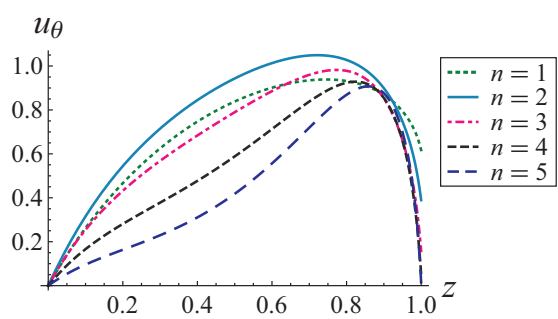

(c)

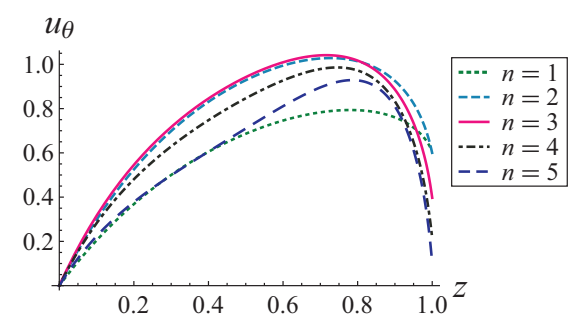

FIG. 6. (Color online) Displacement $u_{\theta}$ against depth $z$ beneath the contact line $r=R$ for (a) $\Lambda=1 / 2$, (b) $\Lambda=1$, and (c) $\Lambda=3 / 2$, as it depends upon the wave number $n$ for $\Upsilon=0.01$. The solid line denotes the critical disturbance $n_{c}$. 
(a)

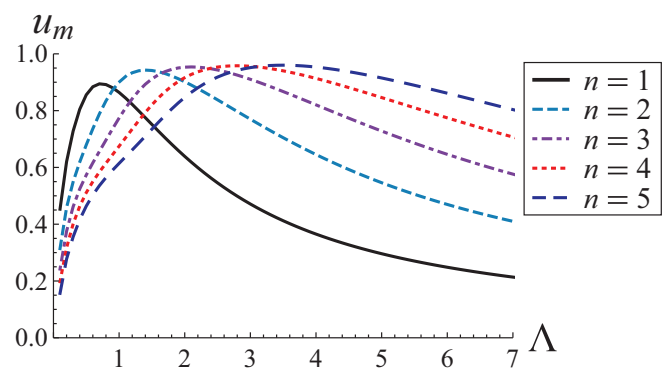

(b)

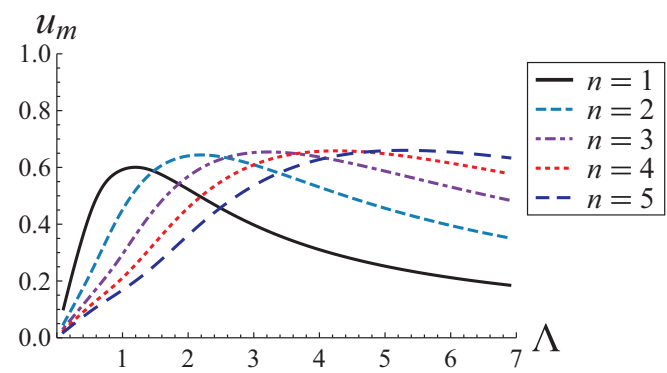

FIG. 7. (Color online) Maximum displacement $u_{m}$ against aspect ratio $\Lambda$ for (a) $\Upsilon=0.1$ and (b) $\Upsilon=1$.

Figure 11 plots the critical wave number $n_{c}$ against the unscaled contact-line radius $R$ for various values of substrate height $h$. As shown, the critical wave number increases (decreases) with decreasing (increasing) substrate height $h$. This result, in effect, implies that large substrate heights are better able to mitigate the imposed surface tractions. Experiments conducted on substrates of three different thicknesses confirm this prediction. Figure 11(b), top and bottom rows, shows three identical experiments conducted on $0.08 \% \mathrm{w}$ agar substrates, but differing thickness, $h=6 \mathrm{~mm}$ (left), $h=20 \mathrm{~mm}$ (center) and $h=40 \mathrm{~mm}$ (right), whose critical wavenumber is $n=5$ (left), $n=2$ (center) and $n=0$ (right), respectively, demonstrating that the thickness of the elastic layer is important in identifying the critical wave number.

Finally, we note that for a given wave number $n$ there is a preferred contact-line radius $\Lambda_{m}$ that generates the largest elastic response $u_{m}^{*}$, as shown in Fig. 7. In Fig. 12, we plot the location $\Lambda_{m}$ [Fig. 12(a)] and value $u_{m}^{*}$ [Fig. 12(b)] of the maximal disturbance as a function of the wave number $n$, as it depends upon the solid elastocapillary number $\Upsilon$. First, we note that the location $\Lambda_{m}$ is an increasing function of both wave number $n$ and solid elastocapillary number $\Upsilon$. In contrast, Fig. 12(b) shows that $u_{m}^{*}$ is a slightly increasing function of $n$ that plateaus for large wave numbers. More specifically, the increase in $u_{m}^{*}$ is particularly evident in Fig. 7(b) when comparing the $n=1$ to the $n=2,3, \ldots$ disturbances. This implies that if, in experiments, one observes $n$ fractures, then it is always possible to adjust the droplet volume $V$ to generate

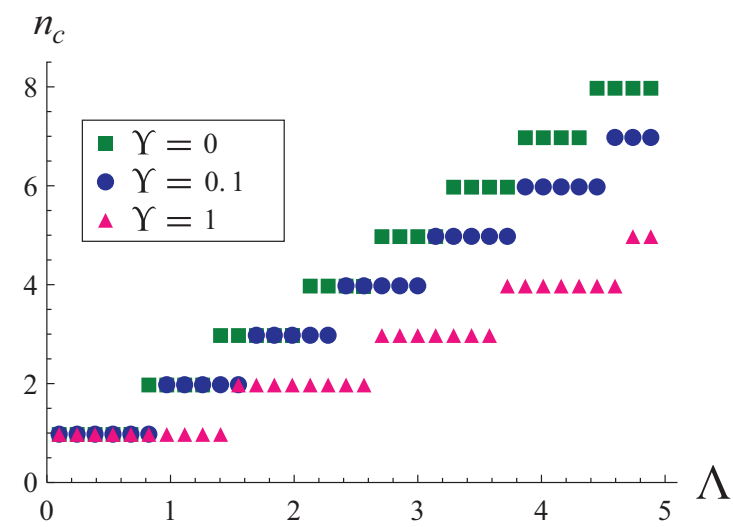

FIG. 8. (Color online) Critical wave number $n_{c}$ against aspect ratio $\Lambda$, as it depends upon $\Upsilon$. $n-1, n-2, \ldots$ fractures. The converse is not necessarily true. Finally, we show that $u_{m}^{*}$ decreases with $\Upsilon$, reflecting the energetic penalty for flexure associated with the solid surface tension. In the large- $\Upsilon$ regime, the gel substrate behaves more like a liquid than a solid. We note that this result is not inconsistent with larger deformations for weaker substrates, as the real displacements are scaled with the elastocapillary length, (16). That is, decreasing the elastic modulus $E$ increases the solid elastocapillary number $\Upsilon$, but also the elastocapillary length $\ell$ and, thus, the real displacements.

We end this section with a comment on the failure criterion. The results presented in this section have assumed that the substrate is always weak enough to generate deformations that exceed the failure threshold for agar gel. As such, we have focused our study on identifying a critical wave number and not the failure threshold. Additional experiments would be needed to extract such information.

\section{CRACK NUCLEATION}

The model developed above captures the central feature required to generate a starburst with a particular number of arms: a critical wave number $n_{c}$ for which the tangential displacement is maximal. In experiments, we observe that for a given set of experimental conditions, a narrow range of wave numbers is selected; this is not surprising given that

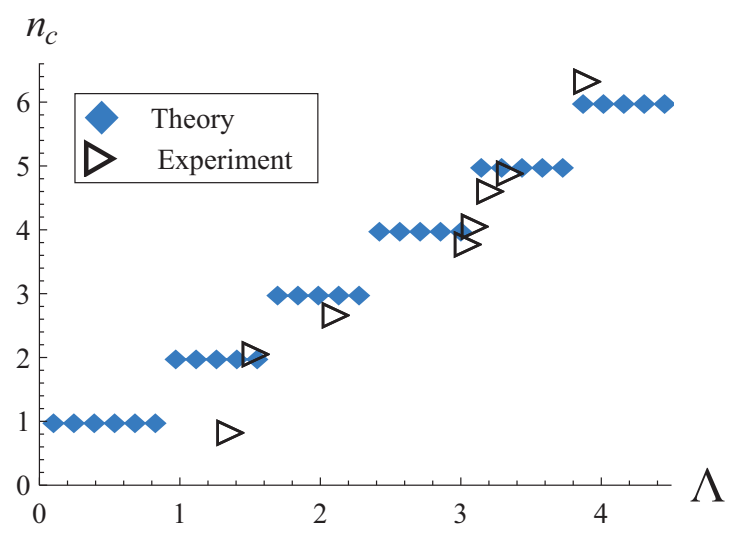

FIG. 9. (Color online) Comparison to experiments by Daniels et al. [14]: critical wave number $n_{c}$ against aspect ratio $\Lambda$ for $\Upsilon=0.1$. Each triangle is the average of several experiments with $\Lambda$ (equivalently, $R, \alpha$ ) estimated using reasonable values. 


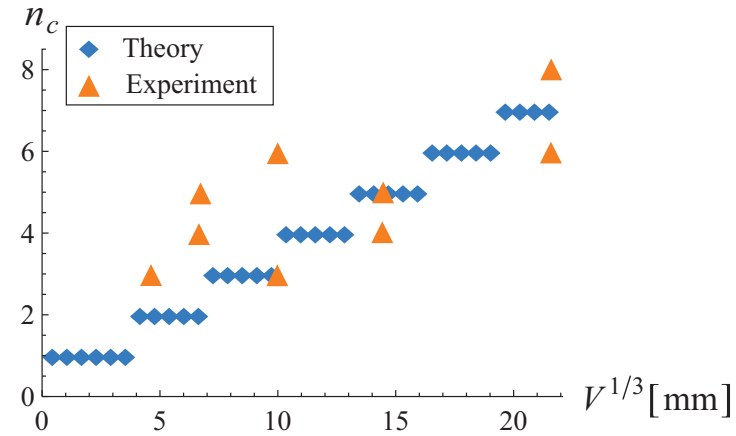

FIG. 10. (Color online) Critical wave number $n_{c}$ against $V^{1 / 3}$ (mm) for $\alpha=83^{\circ}, \Upsilon=0.1$, and $h=5 \mathrm{~mm}$. Each triangle is a single experiment.

values of $n \approx n_{c}$ can have a maximum displacement similar to $n_{c}$. However, the failure criterion does not provide a failure mechanism. In physical gels such as agar and gelatin, the stiffness of the material is provided by a network of entangled polymers [21]. Under strain, these polymers are stretched farther away from equilibrium, although not necessarily to the point of failure. Because polymers are subject to thermal

(a)

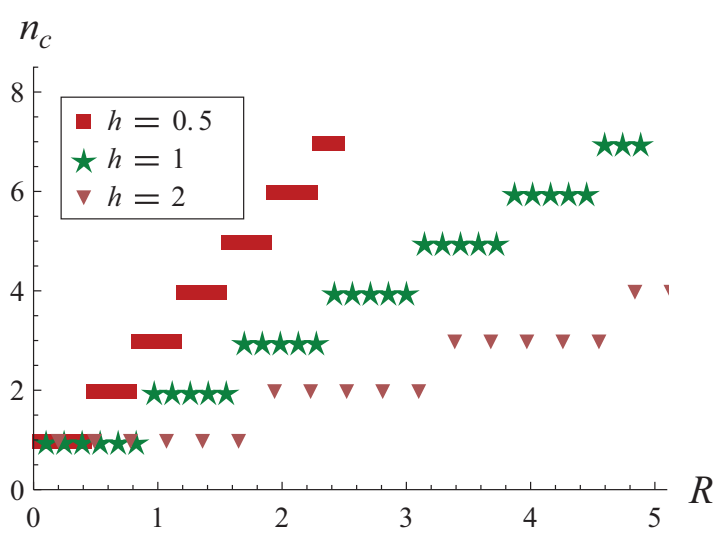

(b)
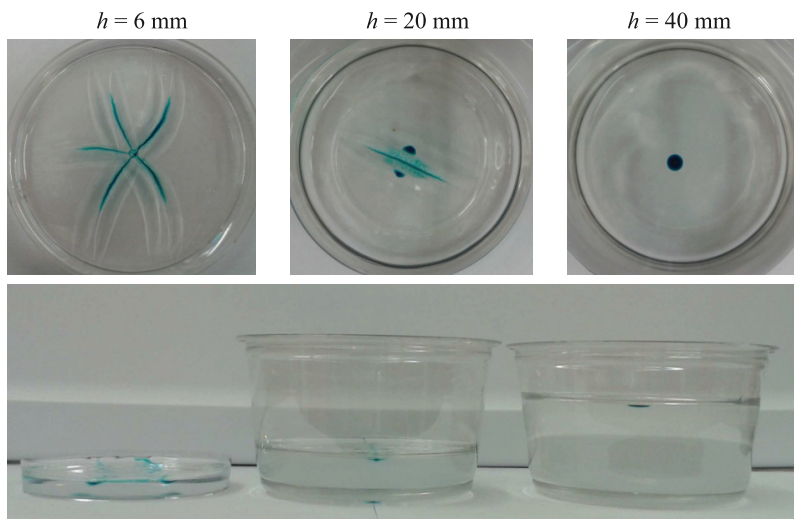

FIG. 11. (Color online) Depth dependence: (a) critical wave number $n_{c}$ against contact-line radius $R$ as a function of the height $h$ of the elastic layer and (b) sample experiments showing typical results for droplets deposited on $0.08 \%$ w agar substrate, with three substrate thicknesses. (a)

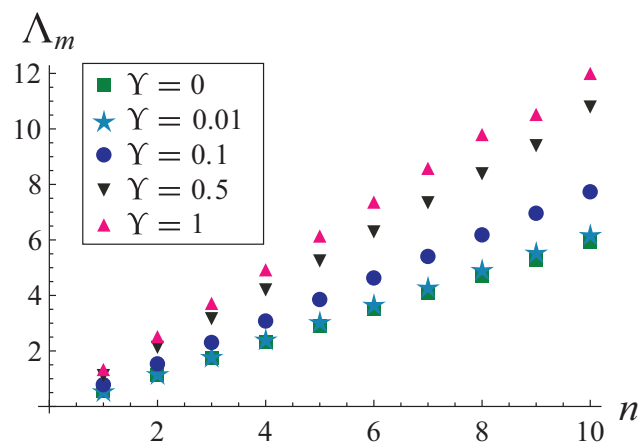

(b)

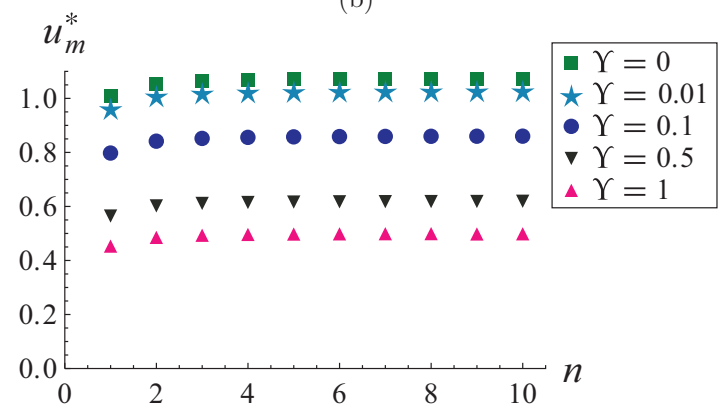

FIG. 12. (Color online) Maximal disturbance: (a) aspect ratio $\Lambda_{m}$ and (b) displacement $u_{m}$ against wave number $n$ as a function of elastocapillary number $\Upsilon$.

fluctuations, even a subcritical strain can be triggered by a sufficiently large thermal fluctuation. This is the mechanism behind delayed fracture, whereby there is an exponential distribution of waiting times (indicating a Poisson process) before which a strained material fractures. This has been directly observed both in stiffer gelatin rods $(E=50 \mathrm{kPa})$ [17] and in vibration-controlled experiments in this system.

\section{CRACK PROPAGATION}

Once a fracture is initiated, fluid begins to flow into the crack tip and the crack propagates outward (away from the central droplet) until the reservoir of fluid is exhausted. We develop a two-dimensional model for the asymptotic propagation of a Mode I fracture driven by a pressure head $\triangle P$. A schematic is shown in Fig. 13. To begin, we consider an incipient crack of length $L(t)$, filled with an inviscid fluid of density $\rho$, propagating into an elastic solid. In this section, we model the solid as either (i) a linear elastic material or (ii) an

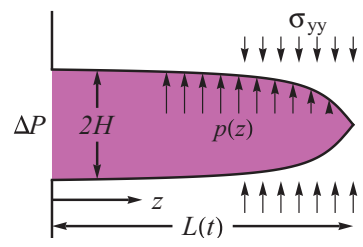

FIG. 13. (Color online) Definition sketch of a fluid-filled crack of length $L$ driven by a pressure head $\Delta P$. 
incompressible neo-Hookean material, as is typically used for polymeric gels, and contrast the computed propagation rates.

We assume that the capillary pressure head $\Delta P=2 \sigma_{l g} / R$ at the fluid reservoir $z=0$ is greater than the elastic stress in the solid in front of that reservoir, so as to drive fluid into the crack tip. The flow $v$ of this inviscid fluid is governed by Bernoulli's law,

$$
\frac{1}{2} \rho v^{2}+p=C
$$

and, hence, driven by the gradient in fluid pressure $p$, which is related to the elastic stress induced by deformations of the crack surface. Here we assume that there is no ambient stress field in the elastic solid or the substrate is not prestressed. The localized stress (pressure) field near the crack tip $z \rightarrow L(t)$ of a linear elastic solid has the well-known asymptotic form [22]

$$
\sigma_{y y}=-p=\frac{K_{I}}{\sqrt{2 \pi}} \frac{1}{\sqrt{L(t)-z}} .
$$

For an incompressible neo-Hookean solid, the asymptotic stress field is given by $[23,24]$

$$
\sigma_{y y}=-p=\frac{K_{I}^{*}}{\sqrt{2 \pi}} \frac{1}{(L(t)-x)^{2 / 3}} .
$$

Here $K_{I}$ and $K_{I}^{*}$ are stress intensity factors for a linear elastic and incompressible neo-Hookean solid, respectively. In general, $K_{I}$ is a function of the crack geometry and far-field loading conditions, but we may simplify our model by utilizing several experimental observations.

Recent experiments have demonstrated self-healing behavior of fluid-filled cracks in soft substrates [15,16]. This observation is characteristic of stable, or transport-limited, crack growth. That is, the speed of crack growth is limited by the extent to which the fluid can be supplied (transported) to the crack tip. For reference, unstable crack growth would be controlled by the speed of an elastic wave in the solid, a characteristic which is not observed experimentally. Hereafter, we assume that the crack propagates in the marginal state $K_{I}=K_{I c}$, where $K_{I c}$ is the fracture toughness, consistent with stable crack growth.

We apply Bernoulli's law, (25), using the crack tip (state 1) and a point upstream (state 2 ) to derive an equation relating the crack-tip velocity $v=\dot{L}$ to the pressure gradient in the linear elastic solid (26),

$$
\dot{L}=\left(\frac{2}{\pi}\right)^{1 / 4} \sqrt{\frac{K_{I c}}{\rho}}\left(\frac{1}{L}\right)^{1 / 4},
$$

whose solution gives

$$
L \propto\left(\frac{K_{I c}}{\rho}\right)^{2 / 5} t^{4 / 5} .
$$

This predicts a characteristic exponent of $\beta=4 / 5$ for the growth of the arm. A similar relationship is derived for an incompressible neo-Hookean solid by applying the respective stress field (27) instead:

$$
\dot{L}=\left(\frac{2}{\pi}\right)^{1 / 4} \sqrt{\frac{K_{I c}^{*}}{\rho}}\left(\frac{1}{L}\right)^{1 / 3} .
$$

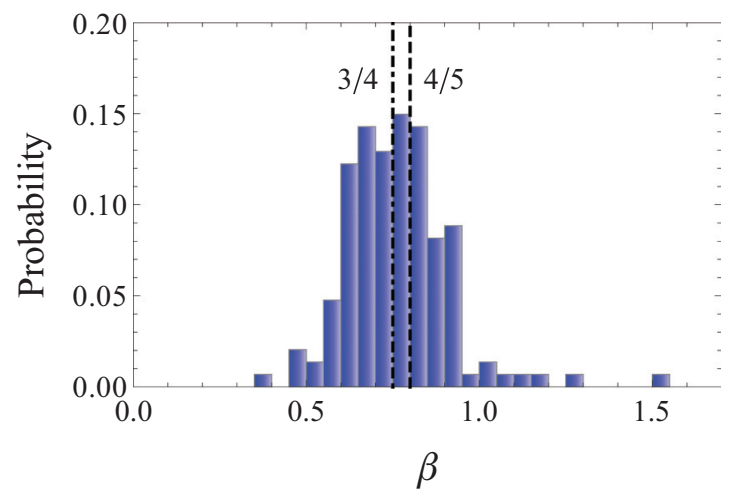

FIG. 14. (Color online) Histogram for the crack length exponent $\beta$ with mean $\langle\beta\rangle=0.76$.

For this system, the characteristic exponent is instead $\beta=3 / 4$ :

$$
L \propto\left(\frac{K_{I c}^{*}}{\rho}\right)^{3 / 8} t^{3 / 4} .
$$

Note that both constitutive models predict power law behavior, but the neo-Hookean model is able to reproduce the universal exponent $t^{3 / 4}$ observed experimentally [14]. We attribute this result to the observation that the gel substrate is incompressible and that the large-scale deformation of the soft solid are more aptly described by the nonlinear neo-Hookean constitutive law.

Because the gels are spatially heterogeneous, cracks nucleate with different widths and propagate at varying rates. We fit the length of each fracture arm to the form $L(t)=A t^{\beta}$ and examine the distribution of $\beta$ values observed. No systematic variation with material parameters was found for the values of $\beta$, but the width of the crack determines the pre-factor $A$ [14]. For this same data set of 147 individual arms, we find that $\beta=$ $0.76 \pm 0.01$ (standard error). The full probability distribution is shown in Fig, 14, which allows for comparison to the $3 / 4$ (neo-Hookean) and 4/5 (linear elastic) constitutive models.

\section{DISCUSSION}

We have analyzed the deformations of a soft elastic substrate induced by the liquid-solid interactions with a liquid droplet, as well as the propagation of a fluid-filled crack in a soft elastic medium. The deformations associated with a corrugated contact-line yield a critical disturbance with wave number $n_{c}$ that generates the largest elastic response within the substrate. We quantify the response with the tangential displacement, a failure criterion which we correlate with the initiation of a starburst fracture (see Fig. 1). Computations reveal that the location of the unbalanced contact-line force is the most important parameter in wave-number selection. Our theoretical predictions compare favorably to (i) previously reported experiments with fixed-volume droplets [14] and (ii) experiments where the contact angle $\alpha$ is fixed and the droplet volume $V$ is varied, thereby confirming our interpretation of the initiation process. Our model also predicts that the substrate thickness $h$ is also an important parameter in wave number selection, an observation which we see in experiments. For the crack propagation problem, we develop a model by considering the transport of an inviscid fluid into 
the crack tip of either a linear elastic or an incompressible neo-Hookean solid. While both elastic models yield power law growth, the neo-Hookean model predicts that the crack length grows with universal exponent $L \propto t^{3 / 4}$ and does not scale with any material parameters, consistent with experimental observations [14-16].

Studies on fracture necessarily take place on materials which are strong enough to resist fracture until the point when the measurement is made. However, many biological materials are soft enough that conventional fracture measurements are not possible because the material is too weak to support its own weight. The present study suggests that by depositing a droplet of known size and wettability, it could be possible to use starburst fractures to measure the fracture toughness of very soft materials as long as they remain fully supported by the dish. Furthermore, because the growth rate of fractures is sensitive to whether or not the material is linear-elastic, this could also provide a new method for materials characterization.

\section{ACKNOWLEDGMENTS}

The authors are grateful for support from the National Science Foundation under Grant No. DMS-0968258, as well as North Carolina State's Undergraduate Research Office, to Michael Shearer for valuable discussions, and to Mark Schillaci for preliminary experiments useful in formulating the model.
[1] B. Roman and J. Bico, J. Phys.: Condens. Matter 22, 493101 (2010).

[2] T. Kajiya, A. Daerr, T. Narita, L. Royon, F. Lequeux, and L. Limat, Soft Matter 9, 454 (2013).

[3] A. Marchand, S. Das, J. H. Snoeijer, and B. Andreotti, Phys. Rev. Lett. 109, 236101 (2012).

[4] J. H Weijs, B. Andreotti, and J. H. Snoeijer, Soft Matter 9, 8494 (2013).

[5] E. R. Jerison, Y. Xu, L. A. Wilen, and E. R. Dufresne, Phys. Rev. Lett. 106, 186103 (2011).

[6] R. W. Style and E. R. Dufresne, Soft Matter 8, 7177 (2012).

[7] S. Das, A. Marchand, B. Andreotti, and J. H. Snoeijer, Phys. Fluids 23, 072006 (2011).

[8] J. Huang, M. Juszkiewicz, W. H. de Jeu, E. Cerda, T. Emrick, N. Menon, and T. P. Russell, Science 317, 650 (2007).

[9] D. Vella, M. Adda-Bedia, and E. Cerda, Soft Matter 6, 5778 (2010).

[10] B. Davidovitch, R. D Schroll, D. Vella, M. Adda-Bedia, and E. Cerda, Proc. Natl. Acad. Sci. USA 108, 18227 (2011).

[11] S. Jung, P. M. Reis, J. James, C. Clanet, and J. W. M. Bush, Phys. Fluids 21, 091110 (2009).
[12] A. A. Evans, S. E. Spagnolie, D. Bartolo, and E. Lauga, Soft Matter 9, 1711 (2013).

[13] T. Mora and A. Boudaoud, Eur. Phys. J. E: Soft Matter 20, 119 (2006).

[14] K. E. Daniels, S. Mukhopadhyay, P. J. Houseworth, and R. P. Behringer, Phys. Rev. Lett. 99, 124501 (2007).

[15] C. Spandagos, T. B. Goudoulas, P. F. Luckham, and O. K. Matar, Langmuir 28, 7197 (2012).

[16] C. Spandagos, T. B. Goudoulas, P. F. Luckham, and O. K. Matar, Langmuir 28, 8017 (2012).

[17] D. Bonn, H. Kellay, M. Prochnow, K. Ben-Djemiaa, and J. Meunier, Science 280, 265 (1998).

[18] X. Wang and W. Hong, Soft Matter 8, 8171 (2012).

[19] T. Young, Phil. Trans. R. Soc. London 95, 65 (1805).

[20] A. Dupré, Théorie Méchanique de La Chaleur (Gauthier-Villars, Paris, 1869).

[21] P. Goldbart and N. Goldenfeld, Phys. Rev. A 39, 1402 (1989).

[22] L. P. Pook, Linear Elastic Fracture Mechanics for Engineers: Theory and Applications (MIT Press, Boston, MA, 2000).

[23] R. A. Stephenson, J. Elast. 12, 65 (1982).

[24] V. R. Krishnan and C.-Y. Hui, Eur. Phys. J. E 29, 61 (2009). 